1

6 Xufang Deng ${ }^{1}$, Yafang Chen ${ }^{2}$, Anna M. Mielech ${ }^{1}$, Matthew Hackbart ${ }^{1}$, Kristina R. Kesely ${ }^{3}$, Robert 7 C. Mettelman ${ }^{1}$, Amornrat O’Brien ${ }^{1}$, Mackenzie E. Chapman ${ }^{2}$, Andrew D. Mesecar ${ }^{2,3}$, and Susan 8 C. Baker ${ }^{1 \#}$

9

${ }^{1}$ Department of Microbiology and Immunology, Loyola University Chicago, Stritch School of

11 Medicine, Maywood, IL, ${ }^{2}$ Department of Biological Sciences, Purdue University, West Lafayette,

12 IN, ${ }^{3}$ Department of Biochemistry, Purdue University, West Lafayette, IN

13

$14 \quad$ \#Corresponding Author: sbaker1@luc.edu

\section{Structure-Guided Mutagenesis Alters Deubiquitinating Activity and Attenuates Pathogenesis of a Murine Coronavirus}

13

15 


\section{Abstract}

Coronaviruses express a multifunctional papain-like protease, termed PLP2. PLP2 acts as a protease that cleaves the viral replicase polyprotein, and a deubiquitinating (DUB) enzyme which removes ubiquitin moieties from ubiquitin-conjugated proteins. Previous in vitro studies implicated PLP2 DUB activity as a negative regulator of the host interferon (IFN) response, but the role of DUB activity during virus infection was unknown. Here, we used X-ray structureguided mutagenesis and functional studies to identify amino acid substitutions within the ubiquitin-binding surface of PLP2 that reduced DUB activity without affecting polyprotein processing activity. We engineered a DUB mutation (Asp1772 to Ala) into a murine coronavirus and evaluated the replication and pathogenesis of the DUB mutant virus (DUBmut) in cultured macrophages and in mice. We found that the DUBmut virus replicates similarly as the wild-type virus in cultured cells, but the DUBmut virus activates an IFN response at earlier times compared to the wild-type virus infection in macrophages, consistent with DUB activity negatively regulating the IFN response. We compared the pathogenesis of the DUBmut virus to the wild-type virus and found that the DUBmut-infected mice had a statistically significant reduction $(\mathrm{p}<0.05)$ in viral titer in livers and spleens at day 5 post-infection, albeit both wild-type and DUBmut virus infections resulted in similar liver pathology. Overall, this study demonstrates that structure-guided mutagenesis aids the identification of critical determinants of PLP2-ubiquitin complex, and that PLP2 DUB activity plays a role as an interferon antagonist in coronavirus pathogenesis.

\section{Importance}

Coronaviruses employ a genetic economy by encoding multifunctional proteins that function in viral replication and also modify the host environment to disarm the innate immune response. The coronavirus papain-like protease 2 (PLP2) domain possesses protease activity, 
which cleaves the viral replicase polyprotein, and also DUB activity (de-conjugating

40 ubiquitin/ubiquitin-like molecules from modified substrates) using identical catalytic residues. To

41 separate the DUB activity from the protease activity, we employed a structure-guided mutagenesis

42 approach and identified residues that are important for ubiquitin-binding. We found that mutating

43 the ubiquitin-binding residues results in a PLP2 that has reduced DUB activity but retains protease

44 activity. We engineered a recombinant murine coronavirus to express the DUB mutant and showed

45 that the DUB mutant virus activated an earlier type I interferon response in macrophages and

46 exhibited reduced pathogenesis in mice. The results of this study demonstrate that PLP2/DUB is

47 an interferon antagonist and a virulence trait of coronaviruses.

\section{Introduction}

Coronaviruses (CoVs) are members of the order Nidovirales, which includes enveloped viruses

with large ( $\sim 30 \mathrm{~kb})$, positive-sense single-stranded RNA genomes that yield a characteristic nested

51 set of subgenomic mRNAs during replication in the cytoplasm of infected cells $(1,2)$. The genome

52 organization for coronaviruses is highly conserved, with the 5'-most two-thirds of the genome

53 encoding the replicase polyprotein, followed by sequences encoding the canonical structural

54 proteins: spike, envelope, membrane, and nucleocapsid. Many CoVs contain accessory genes,

55 which are interspersed among the genes for the structural proteins. Although these accessory genes

56 are not necessarily required for virus replication and are, in general, not highly conserved within

57 the virus family, many encode proteins that regulate the host response (3). Interestingly,

58 coronavirus replicase proteins, which are highly conserved, can also act as antagonists to block or

59 delay the host innate immune response to infection $(1,4-8)$. That a slew of coronavirus-encoded

60 accessory and non-accessory proteins have been shown to shape the host antiviral response

61 suggests that viral-mediated subversion of host defenses is an important element of infection. Here, 
62 we focus on the viral protease/deubiquitinase (DUB) with the goal of assessing the role of DUB

63 activity in shaping the pathogenesis of mouse hepatitis virus (MHV), a model murine coronavirus. the replicase polyprotein (2, 9-11). Murine coronavirus MHV encodes three proteases: two papain-like proteases (PLP1 and PLP2) and one chymotrypsin-like protease (3CLpro, also termed Mpro). MHV PLP2 is similar to the single papain-like protease (termed PLpro) of Severe Acute Respiratory Syndrome Coronavirus (SARS-CoV) and Middle East Respiratory Syndrome Coronavirus (MERS-CoV) $(9,12-16)$. We and others have revealed that PLP2 or PLpro of multiple coronaviruses, including MHV, are multifunctional, not only capable of cleaving the viral polyprotein but also possessing deubiquitinase (DUB) and deISGylating (de-conjugating ISG15 molecule from modified substrates) activities $(9,12,13,15-27)$. However, it has been challenging to study the effects of a mutated DUB on virus replication and pathogenesis because the protease and DUB activities share the same catalytic site, disruption of which is lethal for virus replication. Therefore, it was necessary for us to identify residue(s) within the MHV protease/DUB-ubiquitin binding surface that can be mutated to result in reduced DUB activity without affecting polyprotein

77 processing. In this report, we describe the X-ray structure-guided identification of such residues 78 and evaluate the replication, IFN antagonistic effect, and pathogenesis of a recombinant DUB 79 mutant MHV.

\section{Results}

81 Structure-guided identification of PLP2 residues interacting with ubiquitin. To investigate

82 the role of viral deubiquitinating activity in coronavirus replication and pathogenesis, we first 
84 preserving the enzyme's deISGylating and protease activities, the latter being necessary for viral

85 replication. X-ray structural studies of SARS- and MERS-CoV papain-like protease/DUBs cocrystalized with ubiquitin modified at the c-terminus with a covalent warhead (aldehyde, 3-bromo-

87 propylamine, or propargylamine) allowed for identification of residues that are important for direct interaction with ubiquitin $(17,18,20)$. Here, we took a slightly different approach and mutated the catalytic cysteine (C1716) of MHV PLP2 to a serine residue and co-crystallized it with free ubiquitin. The X-ray structure of MHV PLP2 (C1716S) in complex with ubiquitin was determined to a resolution of $1.85 \AA$ and an overall $\mathrm{R}_{\text {free }}=19.6 \%$ and $\mathrm{R}_{\text {work }}=15.8 \%$ (PDB: $\left.5 \mathrm{WFI}\right)$. The overall

92 structure of the MHV PLP2-ubiquitin complex is similar to other PLP2/PLpro ubiquitin-bound structures (Fig. 1A). Ubiquitin binds within the palm region and is gripped by the zinc-fingers motif while the C-terminus extends into the active site.

Next, we aligned the primary amino acid sequence of the MHV PLP2 domain with the SARS-CoV and MERS-CoV papain-like protease domains (Fig. 1E). The sequence alignment and X-ray structure of the MHV PLP2-ubiquitin complex were then analyzed in conjunction with the previous structural and mutagenesis studies on SARS- and MERS-CoV to identify candidate residues that could be mutated to render a loss of DUB activity in vitro. From this analysis, we identified three residues (R1803, D1772, F1812) in MHV PLP2 that form direct interactions with ubiquitin (Fig. 1B-D). Two of the side chain guanidinium nitrogens of R1803 form direct hydrogen bonds with the backbone carbonyl oxygen of A46 in ubiquitin (Fig. 1B). The two side chain carboxylate oxygens of D1772 in MHV PLP2 interact with ubiquitin by forming direct bonds with each of the guanidinium nitrogens of R42 and with one of the guanidinium nitrogens of R72 (Fig. 1C). Finally, F1812 forms Van der Waals contacts with the side chains of I44 and V70 and the delta-carbon of R42 of ubiquitin (Fig. 1D). 
Biochemical analysis of PLP2 mutants' activities. To identify a mutant MHV PLP2 enzyme that

108

109

110

111

112

113

114

115

116

117 retains protease activity but exhibits reduced DUB and/or deISGylating activity, we performed site-directed mutagenesis on each of the residues (R1803, D1772, F1812) by changing each to an alanine to disrupt interactions with ubiquitin. Each mutant enzyme was expressed, purified, and tested for its ability to hydrolyze three substrates: z-RLRGG-AMC, Ub-AMC, and ISG15-AMC.

The activity of each mutant enzyme toward each substrate relative to the wild-type enzyme is shown in Fig. 2A. All three mutants retained their ability to hydrolyze the peptide substrate but each mutant had altered specificity toward Ub-AMC and ISG15-AMC substrates. Mutation of F1812 resulted in a substantial decrease in hydrolysis of both Ub-AMC and ISG15-AMC (Class I), whereas mutation of R1803 resulted in loss of activity only toward ISG15-AMC (Class II), and mutation of D1772 resulted in loss of activity only toward Ub-AMC (Class III).

Since one of the primary goals of this study is to understand the contribution of DUB activity to viral replication and pathogenesis, we next focused on quantitating further the effects of the D1772A mutant on the steady-state kinetic parameters of MHV PLP2 toward the three different substrates (Fig. 2B). The RLRGG-AMC peptide substrate is often used as surrogate of the viral polyprotein substrate and the kinetic data in Fig. 2B show that this substrate is still wellrecognized and cleaved by the D1772A mutant. In fact, we observed a small rate enhancement in the catalytic efficiency (i.e., $k_{\mathrm{cat}} / \mathrm{K}_{\mathrm{m}}$ ) compared to the wild-type enzyme.

The Ub-AMC substrate, on the other hand, is poorly recognized and cleaved by the D1772A mutant compared to the wild-type enzyme. The wild-type enzyme normally interacts strongly with $\mathrm{Ub}$-AMC with a $\mathrm{K}_{\mathrm{m}}$ value of $0.67 \mu \mathrm{M}$. However, mutation of D1772 to an alanine significantly disrupts the interaction with ubiquitin, making it impossible to saturate MHV PLP2 
under normal experimental conditions (Fig. 2B). The net result is a significant reduction in the catalytic efficiency $\left(k_{\mathrm{cat}} / \mathrm{K}_{\mathrm{m}}\right)$ compared to the wild-type enzyme, which was the goal of these trials.

The kinetic response of MHV PLP2 toward another substrate, ISG15-AMC, was also determined. ISG15 is an important ubiquitin-like modifier that is upregulated and used to ISGylate host proteins during viral infection. A number of viruses, including coronaviruses, engender ISGylation during infection but the function(s) and importance of this activity are not clear (2830). For MHV, neither the wild-type nor the D1772A mutant PLP2 enzyme can be saturated with ISG15-AMC, suggesting weak binding with this ubiquitin-like modifier (Fig. 2B). Moreover, the R1772A mutation does not disrupt the interaction with ISG15 but in fact enhances it to some degree. A potential explanation for the observed selective disruption of ubiquitin binding stems from our analysis of a primary sequence alignment of ubiquitin and ISG15 and the residues that interact with D1772 (Fig. 2C). The interaction between MHV PLP2 D1772 and the R42 residue in human and mouse ubiquitin is absent in human and mouse ISG15 since this residue is a tryptophan in human and mouse ISG15. Therefore, in line with our observations, D1772A mutation would not be expected to alter ISG15 binding. In contrast to R42, residue R72 is conserved in both ubiquitin and ISG15 and its interaction with MHV PLP2 for ubiquitin is likely weaker than that with ISG15.

\section{PLP2 D1772A mutant exhibits reduced DUB activity and inhibition of IFN response in cell}

culture based assays. The in vitro biochemical studies presented here support the notion that we are able to use a structure-guided mutagenesis to uncouple the DUB enzymatic activity from MHV PLP2 while preserving the peptide hydrolysis and deISGylating activities of PLP2. Next, we focused on comparing the activity of the mutant enzyme to its wild-type counterpart for the ability to remove Flag-tagged-ubiquitin conjugated to host proteins in cultured cells (Fig. 3A). We found 
152 that in cells, wild-type PLP2 exhibits robust DUB activity and removes ubiquitin modifications

153 from multiple cellular proteins. On the other hand, the PLP2-D1772A mutant exhibits reduced

154 DUB activity, similar to that of the previously documented catalytic cysteine to alanine mutant,

155 PLP2-CA (19). To determine if this impaired DUB activity altered the ability of PLP2 to act as an 156 interferon antagonist, we transfected cells with a RIG-I expression plasmid, an interferon157 luciferase reporter construct, and either wild-type or mutant PLP2 plasmid and measured luciferase 158 activity at 18 hours post-transfection. In agreement with previous reports $(13,25,31)$, we find that 159 wild-type PLP2 acts as an interferon antagonist, reducing reporter activity by 50-80\%. In contrast, 160 PLP2-D1772A is unable to significantly reduce interferon activation in this assay despite similar 161 expression levels of the wild-type and mutant versions of the protein (Fig. 3B). We also evaluated 162 the protease activity of the enzymes in cells using two independent trans-cleavage assays and 163 found that the wild-type and DUB-mutant enzymes produce similar levels of cleaved products. 164 These results indicate that the D1772A substitution did not alter protease activity (Fig. 3C and D), 165 in agreement with the in vitro kinetic results described above (Fig. 2). Together, these studies 166 reveal that aspartic acid residue 1772 of MHV-PLP2 is important for DUB activity and interferon 167 antagonism, but not for protease activity.

\section{Recombinant MHV harboring PLP2-D1772A activates an earlier IFN response in bone}

169 marrow-derived macrophages. Since the D1772A substitution did not impact protease activity,

170 we reasoned that we should be able to generate recombinant virus containing this substitution,

171 thereby allowing us to determine if the mutation has any effect on viral replication kinetics and

172 interferon antagonism in the context of the live virus. We engineered the mutant virus via reverse

173 genetics (32), performed full genome sequencing to verify the genotype (2 nucleotide changes at 174 positions 5525 and 5526, resulting in D1772A substitution in the replicase polyprotein), and 
175 designated the virus as DUBmut. Upon evaluating virus replication of the DUBmut virus by

176 performing a growth kinetics experiment in parallel with wild-type virus, we found that the

177 DUBmut virus replicates with essentially identical kinetics as the wild-type in a murine

178 astrocytoma cell line (DBT cells) (Fig. 4A). These results are consistent with previous studies of

179 coronavirus interferon antagonists, which showed in many cell lines that viral-mediated interferon

180 antagonism is not essential for virus replication $(5,6)$. Regarding the other ubiquitin-interacting

181 residues identified in the structural analysis, we attempted to rescue virus with substitutions at the

182 F1812 position, but were unable to recover viable virus. These results indicate that F1812 may

183 play a critical role within the polyprotein during virus replication. We were able to recover virus

184 containing the R1803A substitution, but found that it had no detectable phenotype, which we

185 documented in our previous study (5). Here, we focus our efforts on evaluating replication and 186 pathogenesis of the recovered DUBmut virus.

To determine if the impaired DUB activity of the DUBmut virus had an effect on interferon antagonism, we infected primary bone marrow-derived macrophages (BMDMs) and evaluated viral replication kinetics and levels of interferon mRNA and protein. We observed significant activation of interferon alpha (IFN $\alpha$ ) mRNA expression (Fig. 4A) that is coupled with release of

194 impaired relative to the wild-type virus in BMDMs, as measured by level of nucleocapsid RNA 195 (Fig. 4E) and evaluation of infectious virus particle production over time in the kinetics assay (Fig. 
197 of the DUBmut virus, but that this interferon profile is not associated with reduced production of

198 infectious particles in either DBT cells or BMDMs.

199 Disrupting DUB activity mildly attenuates coronavirus pathogenesis in mice. To

200 complement our in vitro studies, we next sought to determine whether loss of DUB activity and

201 the observed activation of interferon during the DUBmut virus infection in macrophages is

202 associated with an attenuated pathogenesis in mice. To test this, we first inoculated mice using the

203 intracranial infection model to evaluate lethality among WT and DUBmut-infected mice, and

204 found similar weight loss and lethality (data not shown). To investigate the pathogenesis in the

205 liver, we inoculated four- (young) or six-week-old (adult) mice intra-peritoneally with a low dose

$206\left(6 \times 10^{3}\right.$ plaque-forming units $\left.[\mathrm{pfu}]\right)$ or a high dose $\left(6 \times 10^{4} \mathrm{pfu}\right)$ of the designated virus, respectively,

207 and measured viral titer in the liver and spleen at the indicated days post-infection (dpi). We

208 observed similar levels of infectious particles in young mice inoculated with a high dose of virus

209 and adult mice with a low dose at 3 and 5 dpi (data not shown), but detected reduced viral titers in

210 the spleens of young mice infected with a low dose of DUBmut virus at 3 dpi (Fig. 5A) and adult

211 mice inoculated with a high dose at 5 dpi (Fig. 5B). Similar pathology in liver sections was

212 observed at 3 and 5 dpi (Fig. 5C). These results indicate that the DUBmut virus is mildly attenuated

213 compared to the wild-type virus, suggesting that the DUB activity does play a role in MHV

214 pathogenesis and PLP2 is a virulence factor.

\section{Discussion}

In the present study, we aimed to investigate the roles of PLP2 DUB activity during

217 coronavirus infection. Through structure-guided mutagenesis, we identified residues of MHV

218 PLP2 that mediates its interaction with ubiquitin. By mutating the residue Asp-1772 to Ala 
219 (D1772A), we found that the DUB activity of PLP2 was greatly reduced and a recombinant MHV

220 carrying this mutation (DUBmut) activated an earlier IFN response in macrophages. Although we

221 only observed a subtle attenuation of the DUBmut virus in the tested animal models, we

222 demonstrated that PLP2 DUB activity does play a role in suppressing the host immune response

223 and is a virulence trait, strengthening our earlier discoveries on SARS-CoV PLpro (4). We further

224 investigated the differences in the interferon response generated in response to WT versus

225 DUBmut virus in our companion study (Volk et al., submitted), which further supports a role for

226 DUB activity in modulating the interferon response in macrophages.

The structure-guided approach used to generate the DUBmut virus allowed for characterization of three different classes of mutant enzymes: Class I, deficient in both DUB and deISGylating activity; Class II, deficient in deISGylating activity only; and Class III, deficient in DUB activity but competent in protease and deISGylating activity. We utilized three unique biochemical substrates, each with a conjugated fluorescent AMC reporter, to evoke the multifunctional activities of PLP2. Activity against the z-RLRGG-AMC peptide substrate represents the polyprotein processing activity of MHV PLP2, while UB-AMC and ISG15-AMC stimulate

234 the deubiquitinating and deISGylating activities of the enzyme, respectively $(17,34)$. The kinetic 235 data for the D1772A DUBmut hydrolysis of the z-RLRGG-peptide provided in Figure 2B indicate that the polyprotein processing ability of this mutant is likely not affected by the D1772A 237 substitution. The deISGylating ability of the enzyme is also not affected. In contrast, the mutant 238 enzyme's deubiquitinating activity is significantly reduced relative to wild-type, which is most 239 likely due to a lowered binding affinity for ubiquitin as the enzyme could not be saturated with $\mathrm{Ub}-\mathrm{AMC}$ as a substrate. 

emerged novel coronavirus 2019 (nCoV-2019) (35-38). By aligning the X-ray structure of the

244 PLpro domains of SARS-CoV bound to ubiquitin (17) with the sequence of nCoV-2019 PLpro 245 (Fig. 6A) and comparing the differences in their residues with those of MHV PLP2 (Fig. 1E), we 246 observe significant differences in the potential interactions between nCoV-2019 and ubiquitin. For 247 example, we found that the hydrogen bonds between MHV PLP2 R1803 and the carbonyl oxygen of UbA46 (Fig. 1B) are lost in nCoV-2019 and SARS-CoV (Fig. 6B). In contrast, the hydrogen bonds between MHV PLP2 D1772 and Ub residues R42 and R72 (Fig. 1C) are likely preserved with the substitution of E1772 in nCoV-2019 and SARS-CoV PLpro (Fig. 6C). The hydrophobic interactions between Ub residues I44 and V70 and F1812 of MHV PLP2 (Fig. 1D) also appear to be preserved with M1812 of nCoV-2019 and SARS-CoV PLpro (Fig. 6D). The X-ray structures of MHV PLP2 and SARS-CoV PLpro, together with our structural model of nCoV-2019 PLpro, will provide testable hypotheses for the design and mutagenesis of the ubiquitin-binding domain (17) and diubiquitin-binding domains (20) of the recently emerged coronavirus nCoV-2019 PLpro.

We were able to reproduce the enzymatic profile of the purified PLP2-D1772A mutant 257 protein when we expressed it in cell culture (Fig. 3). Therefore, our finding that the DUBmut virus containing the PLP2-D1772A substitution activates an elevated antiviral response in macrophages compared to the wild-type virus, but that this antiviral state results in only mild attenuation of disease in mice relative to WT infection, was unexpected. Previous studies demonstrated that ubiquitin has important roles in both the activation and the attenuation of innate antiviral pathways

262 (39); therefore, we anticipated a more remarkable phenotype for a DUB-mutant virus. We can 263 imagine several possible explanations for our findings. First, it is possible that viral DUB activity 
264 has a relatively minor role in shaping pathogenesis in this system. In fact, our recent studies using

265 SARS-CoV and SARS-related CoVs found that the papain-like protease domain/DUB is a

266 virulence trait that varies among members of the SARS-coronavirus species (4). In that study, we

267 found that replacing the SARS PLP2/DUB domain with a SARS-related PLP2/DUB domain

268 reduced the ability of that virus to antagonize the innate immune response. Together, this previous

269 report in conjunction with the current study support the concept that different PLP2/DUB domains

270 may have distinct effects on antagonism of the innate immune response depending on the virus

271 and the host cell type. Another possibility is that the DUB-mutant virus we generated may not have

272 been sufficiently debilitated in its DUB activity to result in altered pathogenesis. We found that it

273 was difficult to recover viable DUB-mutant viruses; indeed, this D1772A mutant was the sole

274 viable DUB-mutant representative of our many attempts. Because an elevated interferon response

275 was elicited from the DUBmut-infected cells, this mutant virus fulfilled our criteria demonstrating

276 the inactivation of an interferon antagonist. However, we speculate that if we are able to recover

277 mutants that exhibit a range of DUB activity, we may be able to more fully assess the role of DUB

278 activity as a contributor to coronaviral pathogenesis. Despite these caveats, the MHV DUB-mutant

279 generated in this study did exhibit a reproducible phenotype of eliciting an elevated interferon

280 response in infected macrophages that was associated with mild attenuation of pathogenesis with

281 reduced titers in the livers and spleens of infected mice. Overall, we conclude that DUB activity

282 is indeed a virulence trait, and contributes to the ability of MHV to modulate the host innate

283 immune response to infection. Further studies are needed to identify the targets of viral DUB

284 activity and the detailed role of DUB activity in delaying the innate immune response to virus 285 replication.

286 Materials and Methods 


\section{Ethics Statement}

The mouse experiment in this study was carried out in accordance with the recommendations in the Guide for the Care and Use of Laboratory Animals of the National Institutes of Health. The experimental protocol was reviewed and approved by the Institutional Animal Care and Use Committee (IACUC) at Loyola University Chicago (IACUC\#: 2016-029). C57BL/6 female mice were purchased from The Jackson Laboratory and maintained in the Comparative Medicine Facility of Loyola University Chicago. Mice were consistently monitored for signs of distress over the course of the experiments to be removed from the experiment and euthanized using carbon dioxide inhalation to prevent unnecessary suffering.

\section{Cells}

Human embryonic kidney (HEK) 293T cells were purchased the from American Type Culture Collection (ATCC, \# CRL-11268) and maintained in DMEM (\#10-017-CV, Corning) containing $10 \%$ fetal calf serum (FCS) and supplemented with $1 \%$ nonessential amino acids, $1 \%$ HEPES, 2\% L-glutamine, 1\% sodium pyruvate, and 1\% penicillin/streptomycin. DBT cells were cultured in MEM (\#61100-061, ThermoFisher) supplemented with 5\% FCS, 2\% L-glutamine, and 10\% Tryptose Phosphate Broth (TPB). The 17Cl-1 cell line was maintained in DMEM containing 5\% FCS. Baby hamster kidney cells expressing the MHV receptor (BHK-R) were kindly provided by Mark Denison (Vanderbilt University Medical Center) and maintained in DMEM supplemented with $10 \%$ FCS, $2 \%$ L-glutamine, and $0.8 \mu \mathrm{g} / \mathrm{mL}$ G418. Bone marrow-derived macrophages (BMDMs) were prepared and cultured as described previously (5).

\section{Plasmids and mutagenesis}

The sequence of the PLP2 domain (amino acids 1525-1911 of MHV pp1ab) in frame with a V5 epitope tag was codon-optimized, synthesized by Genscript (Piscataway, NJ) (sequence available upon request), and cloned into pCAGGS vector. For mutagenesis, an overlapping PCR 
311

312

313

314

315

316

317

318

319

320

321

322

323

324

325

326

327

328

329

330

331

332

333

strategy was used with synthetic primers (sequences available upon request). The introduced mutations were verified by sequencing. The RIG-I and nsp2/3-GFP expression plasmid was kindly provided Ralph Baric (University of North Carolina). The IFN $\beta$-Luc reporter plasmid was a gift of John Hiscott (Jewish General Hospital, Montreal, Canada). The Flag-Ub plasmid was kindly provided by Adriano Marchese (University of Wisconsin-Milwaukee).

\section{MHV PLP2 wild-type and mutant purification, kinetics, and X-ray structure}

The wild-type, C1716S, D1772A, R1803A, and F1812A mutant enzymes were expressed and purified similar to our previously published methods except that the MHV PLP2 construct used here (amino acids N1609 to N1909) was absent the DPUP domain (16). Crystallization and X-ray structure determination details will be published elsewhere. Steady-state kinetic studies on the wild-type, D1772A, R1803A, and F1812A mutant enzymes with substrates Z-RLRGG-AMC (where $\mathrm{Z}$ is a carboxybenzyl protecting group), Ubiquitin-AMC, and ISG15-AMC were performed as described previously (16). Structure figures were generated with the software program UCSF Chimera (40).

\section{Protease and deubiquitinating activity assays}

To determine the protease activity of PLP2, HEK293T cells grown to 70\% confluency in 24-well plates (Corning) were transfected using TransIT-LT1 (MIR2300, Mirus) according to the manufacturer's protocol. For the protease activity assay, HEK293T cells were transfected with 25 ng nsp2/3-GFP plasmid and 200 ng pCAGGS-PLP2-V5 expression plasmids (wild-type and mutant). To determine deubiquitinating activity of the proteins, cells were transfected with $200 \mathrm{ng}$ Flag-Ub plasmid and pCAGGS-PLP2-V5 expression plasmids (wild-type and mutant). Cells were lysed $24 \mathrm{~h}$ post-transfection with $100 \mu \mathrm{L}$ of lysis buffer (comprising $20 \mathrm{mM}$ Tris [pH 7.5], 150 mM NaCl, 1mM EGTA, 1mM EDTA, 1\% Triton X-100, 2.5 mM sodium pyrophosphate, 1mM $\beta$ - 
334 glycerophosphate, $1 \mathrm{mM}$ sodium orthovanadate, $1 \mu \mathrm{g} / \mathrm{mL}$ leupeptin, and $1 \mathrm{mM}$

335 phenylmethylsulfonyl fluoride). Whole cell lysates were separated by SDS-PAGE and transferred

336 to PVDF membrane in transfer buffer (0.025M Tris, $0.192 \mathrm{M}$ glycine, $20 \%$ methanol) for 1 hour

337 at 60 Volts at $4{ }^{\circ} \mathrm{C}$. Following this, the membrane was blocked using 5\% dried skim milk in TBST

338 buffer $(0.9 \% \mathrm{NaCl}, 10 \mathrm{mM}$ Tris- $\mathrm{HCl}, \mathrm{pH} 7.5,0.1 \%$ Tween 20$)$ overnight at $4^{\circ} \mathrm{C}$. The membrane

339 was incubated with either polyclonal rabbit anti-GFP antibody (A11122, Life Technologies) for

340 the protease assay, or mouse anti-flag (F3165, Sigma) for the DUB assay. The membrane was then

341 washed three times for 15 minutes in TBST buffer followed by incubation with either secondary

342 donkey anti-rabbit-HRP antibody (711-035-152, Jackson ImmunoResearch) or goat anti-mouse-

343 HRP antibody (1010-05, SouthernBiotech). Then the membrane was washed three more times for

34415 minutes in TBST buffer. Detection was performed using Western Lighting Chemiluminescence

345 Reagent Plus (PerkinElmer) and visualized using a FluoroChemE Imager (Protein Simple). The

346 expression of PLP2, $\beta$-actin, and calnexin were probed with mouse anti-V5 antibody (R960,

347 ThermoFisher), mouse anti- $\beta$-actin (A00702, Genscript), or mouse anti-calnexin antibody (2433S,

348 Cell Signaling), respectively.

349

350

351

352

353

354

\section{Biosensor live cell assay}

The protease activity of PLP2 was also assessed using a biosensor live cell assay as described previously (14). Briefly, HEK293T cells in a 96 black-wall plate were transfected with 37.5 ng pGlo-RLKGG construct and 50 ng PLP2 expression plasmids. GloSensor (E1290, Promega) reagent diluted in DMEM+10\% FCS was added at 18 hpi. Plates were read using a luminometer (Veritas) every hour over a course of 5 hours. 


\section{Generating DUB-mutant MHV}

We used a previously described reverse genetics system of MHV-A59 (32) to generate the DUB-mutant virus. Briefly, the nucleotides coding for the Asp-1772 of the PLP2 domain were mutated via site-directed mutagenesis. Viral genomic RNA from in vitro transcription of ligated cDNA fragments using a mMESSAGE mMACHINE T7 Transcription Kit (AM1344, Thermal Fisher) was electroporated into BHK-R cells. Cell supernatants were collected as viral stock following observation of cytopathic effects. Rescued virus was plaque-purified, propagated on BHK-R cells, and titrated on $17 \mathrm{Cl}-1$ cells. The stock virus was subjected to full-genome sequencing and the sequences were aligned to the parental strain, with the intended engineered mutation detected and no additional mutations detected (Kansas State University Diagnostic Laboratory).

\section{Growth kinetics}

DBT cells or BMDMs were infected with wild-type icMHV-A59 or DUB-mutant virus at a multiplicity of infection (MOI) of 1 in serum-free medium. After a one-hour incubation, the inoculum was replaced with fresh, complete medium. Cell culture supernatants were collected at indicated time points and titrated by plaque assay on $17 \mathrm{Cl}-1$ cells. Titers were obtained from three independent assays for each sample. Graphs of virus kinetics were generated using Prism software (GraphPad Software).

\section{Quantification of IFN $\alpha$ production by RT-qPCR and ELISA}

BMDMs in a 12-well plate were mock-infected or infected with MHV at a MOI of 1 . At indicated time points, monolayer cells were collected for RNA extraction and cell culture supernatants were harvested for ELISA analysis. To determine IFN- $\alpha 11$, $\beta$-actin, or MHV-A59 N gene mRNA levels, total RNA was extracted from collected cells using a RNeasy Mini Kit (74104, 
379 Strand Kit (330401, Qiagen). qPCR was then performed with specific primers for mouse IFN- $\alpha 11$ (PPM03050B-200, Qiagen), mouse $\beta$-actin (PPM02945B-200, Qiagen), or MHV-A59 N gene using RT2 SYBR Green qPCR Mastermix (330502, Qiagen) in the Bio-Rad CFX96 system. The thermocycler was set as follows: one step at $95^{\circ} \mathrm{C}(10 \mathrm{~min}), 40$ cycles of $95^{\circ} \mathrm{C}(15 \mathrm{~s}), 60^{\circ} \mathrm{C}(1 \mathrm{~min})$ and plate read, one step at $95{ }^{\circ} \mathrm{C}(10 \mathrm{~s})$, and a melt curve from $65^{\circ} \mathrm{C}$ to $95^{\circ} \mathrm{C}$ at increments of $0.5^{\circ} \mathrm{C} / 0.05 \mathrm{~s}$. Samples were evaluated in triplicate and data are representative of three independent

$\left[\Delta C_{T}=C_{T(\text { gene of interest })}-C_{T(\beta \text {-actin })}\right]$. The secreted amount of IFN- $\alpha$ in culture supernatants was assayed using a mouse IFN- $\alpha$ ELISA kit (BMS6027, eBioscience) according to the manufacturer's instructions.

389 Facility at Loyola University Chicago.

\section{Mouse experiments}

\section{Acknowledgments}

Evaluation of MHV pathogenesis in laboratory mouse was previously described $(5,41)$. Briefly, for intracranial infections, six-week-old C57BL/6 female mice were inoculated with 600 pfu of virus in $20 \mu \mathrm{L}$ PBS. Infected mice were monitored for body weight daily and euthanized when weight loss surpassed $25 \%$. Statistical analyses of survival rate were performed using the log-rank test. For intraperitoneal infection, six- or four week-old mice were injected with $6 \times 10^{3}$ or $6 \times 10^{4}$ pfu of virus in $100 \mu \mathrm{L}$ PBS. Organs were collected at indicated time points and evaluated for viral burden. Liver pathology was evaluated using H\&E staining by the Tissue Processing Core

This work was supported by the National Institutes of Health (NIH) grant R01 AI085089

(to SCB and ADM). MH and RCM were supported by NIH T32 Training Grant for Experimental Immunology (\#AI007508) and RCM was supported by the Arthur J. Schmitt Dissertation 
402 Fellowship in Leadership and Service (Arthur J. Schmitt Foundation). MEC was supported by an

403 NIH/NIGMS T32 Training Grant for Structural Biology and Biophysics (\#GM132024).

404 Crystallization and DNA sequencing were partially supported by the Purdue Center for Cancer

405 Research Macromolecular Crystallography and DNA Sequencing Shared Resources, which are 406 supported by NIH grant P30 CA023168.

407 Author Contributions

Conceptualization: XD, ADM and SCB. Investigation: XD, YC, AMM, MH, KRK, RCM,

409 MH, AO, MEC. Formal Analysis: XD, YC, AMM, MH, KRK, RCM, AO, ADM and SCB. Writing

410 - Original Draft Preparation: XD, YC, AMM, ADM and SCB. Writing - Review \& Editing: with

411 comments from XD, YC, AMM, MH, KRK, RCM, MH, AO, ADM and SCB. Visualization: XD,

412 YC, KRK, AO, MEC, ADM and SCB. Funding Acquisition and Supervision: ADM and SCB.

413 References

414 1. Perlman S, Netland J. 2009. Coronaviruses post-SARS: update on replication and 415 pathogenesis. Nat Rev Microbiol 7(6):439-50.

416 2. Enjuanes L, Almazán F, Sola I, Zuñiga S. 2006. Biochemical aspects of coronavirus 417 replication and virus-host interaction. Annu Rev Microbiol 60:211-230.

418 3. Totura AL, Baric RS. 2012. SARS coronavirus pathogenesis: host innate immune 419 responses and viral antagonism of interferon. Curr Opin Virol 2:264-75.

420 4. Niemeyer D, Mösbauer K, Klein EM, Sieberg A, Mettelman RC, Mielech AM, Dijkman

421 R, Baker SC, Drosten C, Müller MA. 2018. The papain-like protease determines a 422 virulence trait that varies among members of the SARS-coronavirus species. PLOS Pathog $423 \quad 14: \mathrm{e} 1007296$. 
424 5. Deng X, Hackbart M, Mettelman RC, O’Brien A, Mielech AM, Yi G, Kao CC, Baker SC.

425

426

427

428

429

430

431

432

433

434

435

436

437

438

439

440

441

442

443

444

445

446

2017. Coronavirus nonstructural protein 15 mediates evasion of dsRNA sensors and limits apoptosis in macrophages. Proc Natl Acad Sci U S A 114:E4251-E4260.

6. Kindler E, Gil-Cruz C, Spanier J, Li Y, Wilhelm J, Rabouw HH, Züst R, Hwang M, V'kovski P, Stalder H, Marti S, Habjan M, Cervantes-Barragan L, Elliot R, Karl N, Gaughan C, van Kuppeveld FJM, Silverman RH, Keller M, Ludewig B, Bergmann CC, Ziebuhr J, Weiss SR, Kalinke U, Thiel V. 2017. Early endonuclease-mediated evasion of RNA sensing ensures efficient coronavirus replication. PLOS Pathog 13:e1006195.

7. Menachery VD, Gralinski LE, Mitchell HD, Dinnon KH, Leist SR, Yount BL, McAnarney ET, Graham RL, Waters KM, Baric RS. 2018. Combination attenuation offers strategy for live-attenuated coronavirus vaccines. J Virol 92:e00710-18.

8. Kindler E, Thiel V, Weber F. 2016. Interaction of SARS and MERS Coronaviruses with the Antiviral Interferon Response, p. 219-243. In Advances in virus research.

9. Mielech AM, Chen Y, Mesecar AD, Baker SC. 2014. Nidovirus papain-like proteases: Multifunctional enzymes with protease, deubiquitinating and deISGylating activities. Virus Res 194:184-190.

10. Kanjanahaluethai A, Baker SC. 2000. Identification of mouse hepatitis virus papain-like proteinase 2 activity. J Virol 74:7911-21.

11. Baker SC, Shieh CK, Soe LH, Chang MF, Vannier DM, Lai MM. 1989. Identification of a domain required for autoproteolytic cleavage of murine coronavirus gene A polyprotein. $\mathbf{J}$ Virol 63:3693-9.

12. Barretto N, Jukneliene D, Ratia K, Chen Z, Mesecar AD, Baker SC. 2005. The papain-like protease of severe acute respiratory syndrome coronavirus has deubiquitinating activity. $\mathbf{J}$ 
Virol 79(24):15189-15198.

448 13. Mielech AM, Kilianski A, Baez-Santos YM, Mesecar AD, Baker SC. 2014. MERS-CoV papain-like protease has deISGylating and deubiquitinating activities. Virology 450

14. Kilianski A, Mielech A, Deng X, Baker SC. 2013. Assessing Activity and Inhibition of MERS-CoV Papain-like and 3C-like Proteases Using Luciferase-based Biosensors. J Virol 87(21):11955-11962.

454 15. Zheng D, Chen G, Guo B, Cheng G, Tang H. 2008. PLP2, a potent deubiquitinase from murine hepatitis virus, strongly inhibits cellular type I interferon production. Cell Res

16. Chen Y, Savinov SN, Mielech AM, Cao T, Baker SC, Mesecar AD. 2015. X-ray structural and functional studies of the three tandemly linked domains of non-structural protein 3 (nsp3) from murine hepatitis virus reveal conserved functions. J Biol Chem 290:25293-

17. Ratia K, Kilianski A, Baez-Santos YM, Baker SC, Mesecar A. 2014. Structural basis for the ubiquitin-linkage specificity and deISGylating activity of SARS-CoV papain-like protease. PLoS Pathog 10:e1004113.

464 18. Bailey-Elkin BA, Knaap RCM, Johnson GG, Dalebout TJ, Ninaber DK, van Kasteren PB, Bredenbeek PJ, Snijder EJ, Kikkert M, Mark BL. 2014. Crystal structure of the MERS coronavirus papain-like protease bound to ubiquitin facilitates targeted disruption of deubiquitinating activity to demonstrate its role in innate immune suppression. J Biol Chem 289:34667-82.

19. Mielech AM, Deng X, Chen Y, Kindler E, Wheeler DL, Mesecar AD, Thiel V, Perlman S, 
Baker SC. 2015. Murine coronavirus ubiquitin-like domain is important for papain-like protease stability and viral pathogenesis. J Virol 89:4907-4917.

472 20. Békés M, van der Heden van Noort GJ, Ekkebus R, Ovaa H, Huang TT, Lima CD. 2016.

473 Recognition of Lys48-Linked Di-ubiquitin and Deubiquitinating Activities of the SARS

$474 \quad$ Coronavirus Papain-like Protease. Mol Cell 62:572-585.

475 21. Bailey-Elkin BA, Knaap RCM, Kikkert M, Mark BL. 2017. Structure and Function of $476 \quad$ Viral Deubiquitinating Enzymes. J Mol Biol 429:3441-3470.

477 22. Clasman JR, Báez-Santos YM, Mettelman RC, O’Brien A, Baker SC, Mesecar AD. 2017. X-ray Structure and Enzymatic Activity Profile of a Core Papain-like Protease of MERS Coronavirus with utility for structure-based drug design. Sci Rep 7:40292.

480

481

482

483

484

485

486

487

488

489

490

491

492

23. Lindner HA, Fotouhi-Ardakani N, Lytvyn V, Lachance P, Sulea T, Ménard R. 2005. The papain-like protease from the severe acute respiratory syndrome coronavirus is a deubiquitinating enzyme. J Virol 79(24):15199-208.

24. Chen Z, Wang Y, Ratia K, Mesecar AD, Wilkinson KD, Baker SC. 2007. Proteolytic processing and deubiquitinating activity of papain-like proteases of human coronavirus NL63. J Virol 81(11):6007-18.

25. Clementz M, Chen Z, Banach BS, Wang Y, Sun L, Ratia K, Baez-Santos YM, Wang J, Takayama J, Ghosh AK, Li K, Mesecar AD, Baker SC. 2010. Deubiquitinating and interferon antagonism activities of coronavirus papain-like proteases. J Virol 84(9):4619_ 29.

26. Xing Y, Chen J, Tu J, Zhang B, Chen X, Shi H, Baker SC, Feng L, Chen Z. 2013. The papain-like protease of porcine epidemic diarrhea virus negatively regulates type I interferon pathway by acting as a viral deubiquitinase. J Gen Virol 94:1554-67. 
27. Deng X, Agnihothram S, Mielech AM, Nichols DB, Wilson MW, StJohn SE, Larsen SD, Mesecar AD, Lenschow DJ, Baric RS, Baker SC. 2014. A chimeric virus-mouse model system for evaluating the function and inhibition of papain-like proteases of emerging coronaviruses. J Virol 88:11825-33.

28. Frias-Staheli N, Giannakopoulos N V, Kikkert M, Taylor SL, Bridgen A, Paragas J, Richt J a, Rowland RR, Schmaljohn CS, Lenschow DJ, Snijder EJ, García-Sastre A, Virgin HW. 2007. Ovarian tumor domain-containing viral proteases evade ubiquitin- and ISG15dependent innate immune responses. Cell Host Microbe 2(6):404-16.

29. Lenschow DJ, Lai C, Frias-staheli N, Giannakopoulos N V, Lutz A, Wolff T, Osiak A, Levine B, Schmidt RE, Garc1 A, Leib DA, Pekosz A, Knobeloch K, Horak I, Whiting H, Iv V. 2007. IFN-stimulated gene 15 functions as a critical antiviral molecule against influenza, herpes, and Sindbis viruses. Proc Natl Acad Sci USA 104(4):1371-1376.

30. Skaug B, Chen ZJ. 2010. Emerging role of ISG15 in antiviral immunity. Cell 143(2):187190.

31. Devaraj SG, Wang N, Chen Z, Chen Z, Tseng M, Barretto N, Lin R, Peters CJ, Tseng CTK, Baker SC, Li K. 2007. Regulation of IRF-3-dependent innate immunity by the papain-like protease domain of the severe acute respiratory syndrome coronavirus. J Biol Chem 282(44):32208-21.

32. Yount B, Denison MR, Weiss SR, Ralph S, Baric RS. 2002. Systematic assembly of a full-length infectious cDNA of mouse hepatitis virus strain A59. J Virol 76:11065-11078.

33. Roth-Cross JK, Bender SJ, Weiss SR. 2008. Murine coronavirus mouse hepatitis virus is recognized by MDA5 and induces type I interferon in brain macrophages/microglia. $\mathrm{J}$ Virol 82:9829-38. 
34. Báez-Santos YM, Mielech AM, Deng X, Baker S, Mesecar AD. 2014. Catalytic function and substrate specificity of the papain-like protease domain of nsp3 from the Middle East respiratory syndrome coronavirus. J Virol 88:12511-27.

35. Zhu N, Zhang D, Wang W, Li X, Yang B, Song J, Zhao X, Huang B, Shi W, Lu R, Niu P, Zhan F, Ma X, Wang D, Xu W, Wu G, Gao GF, Tan W. 2020. A Novel Coronavirus from Patients with Pneumonia in China, 2019. N Engl J Med NEJMoa2001017.

36. Huang C, Wang Y, Li X, Ren L, Zhao J, Hu Y, Zhang L, Fan G, Xu J, Gu X, Cheng Z, Yu T, Xia J, Wei Y, Wu W, Xie X, Yin W, Li H, Liu M, Xiao Y, Gao H, Guo L, Xie J, Wang G, Jiang R, Gao Z, Jin Q, Wang J, Cao B. 2020. Clinical features of patients infected with 2019 novel coronavirus in Wuhan, China. Lancet 0.

37. Zhou P, Yang X-L, Wang X-G, Hu B, Zhang L, Zhang W, Si H-R, Zhu Y, Li B, Huang CL, Chen H-D, Chen J, Luo Y, Guo H, Jiang R-D, Liu M-Q, Chen Y, Shen X-R, Wang X, Zheng X-S, Zhao K, Chen Q-J, Deng F, Liu L-L, Yan B, Zhan F-X, Wang Y-Y, Xiao GF, Shi Z-L. 2020. A pneumonia outbreak associated with a new coronavirus of probable bat origin. Nature 1-4.

38. Wu F, Zhao S, Yu B, Chen Y-M, Wang W, Song Z-G, Hu Y, Tao Z-W, Tian J-H, Pei YY, Yuan M-L, Zhang Y-L, Dai F-H, Liu Y, Wang Q-M, Zheng J-J, Xu L, Holmes EC, Zhang Y-Z. 2020. A new coronavirus associated with human respiratory disease in China. Nature 1-8.

39. Heaton SM, Borg NA, Dixit VM. 2016. Ubiquitin in the activation and attenuation of innate antiviral immunity. J Exp Med 213:1-13.

40. Pettersen EF, Goddard TD, Huang CC, Couch GS, Greenblatt DM, Meng EC, Ferrin TE. 2004. UCSF Chimera?A visualization system for exploratory research and analysis. J 
Comput Chem 25:1605-1612.

540

541

542

543

$544 \quad$ Figure Legends

545

546

547

548

549

550

551

552

553

554

555

556

557

558

559

560

41. Deng X, StJohn SE, Osswald HL, O’Brien A, Banach BS, Sleeman K, Ghosh AK, Mesecar AD, Baker SC. 2014. Coronaviruses resistant to a 3C-like protease inhibitor are attenuated for replication and pathogenesis, revealing a low genetic barrier but high fitness cost of resistance. J Virol 88:11886-11898.

Figure 1. X-ray structure of the MHV PLP2-Ubiquitin complex and residues involved in ubiquitin-binding. (A) Overall structure of MHV PLP2-C1716S-Ub complex. Domains are color coded: Ub in yellow, Ub12 domain of PLP2 in purple, thumb domain in orange, palm domain in cyan, and fingers domain in green. Residue D1772 of PLP2 is located outside of the active site which is circled in black. (B) Hydrogen bond interactions between MHV PLP2 R1803 and the backbone of A46 of ubiquitin. (C) Binding interactions between MHV PLP2 D1772 and two arginine residues (R42 and R72) of ubiquitin. (D) Hydrophobic interactions between F1812 of MHV PLP2 and ubiquitin residues I44 and V70. The 2Fo-Fc maps (blue) surrounding the residues are contoured at $1 \sigma$ in each panel. The PDB coordinates for the MHV PLP2-C1716S-Ub complex have been deposited under PDB Code 5WFI. (E) Sequence alignment (MultAlign) of coronavirus papain-like protease/deubiquitinating domains from MHV (1606-1911 aa, accession \#AAX23975), SARS (1541-1854 aa, accession \#ACZ72209), 2019-nCoV (1564-1878, accession \#QHO60603) and MERS (1480-1803 aa, accession \# AHY21467). Amino acids are colored by similarity using the RISER coloring scheme. Numbering shown is based on MHV sequence. Amino acids mutated in this study are indicated with a black asterisk, the catalytic cysteine is indicated by a blue asterisk, and those amino acids that bind ubiquitin and were mutated in this 

$581 \pm$ SD

study are boxed in green. The active site substrate binding loop also involved in binding inhibitors of SARS is shown highlighted in yellow. The sequence alignment was created using ESPript3.

\section{Figure 2. Structure-guided mutagenesis of MHV PLP2 reveals that D1772A disrupts} ubiquitin binding and reduces DUB activity. (A) Relative kinetic activities of three mutant MHV PLP2 enzymes toward three substrates: z-RLRGG-AMC (green), Ub-AMC (blue), and ISG15-AMC (yellow) compared to the wild-type enzyme. (B) Steady-state kinetic parameters for wild type and D1772A mutant enzymes. (C) Sequence alignment of Ub and ISG15 from human and mouse generated by Clustal Omega. The two arginine residues of Ub (R42 and R72) that interact with D1772 are indicated by arrows. R72 is conserved between Ub and ISG15, whereas R42 (shaded in yellow) is only present in Ub. Accession numbers: Human_Ub, 1ubq; Mouse_Ub, P62991; Human_ISG15, AAH09507; Mouse_ISG15, AAI09347. The sequence alignment was created using ESPript.

Figure 3. D1772A substitution in the coronavirus papain-like protease Ub-binding site reduces DUB activity and interferon antagonism without reducing protease activity. (A) Western blot assessing the DUB activity of PLP2. (B) IFN antagonism of PLP2 was determined using an IFN-luciferase reporter stimulated by N-RIG-I expression. The reporter activity of vector control was set to $100 \%$ (indicated by a dash line). (C and D) Protease activity was evaluated using (C) a trans-cleavage assay that detects the cleaved products by western blot and (D) a pGlo biosensor assay which is activated by PLP2-mediated cleavage of the substrate. Data are representative of at least two independent experiments. Data in (B) and (D) are presented as means

Figure 4. Evaluating the replication kinetics of, and level of interferon activation by, WT MHV and DUBmut in cell culture. (A) Replication kinetics of WT and DUBmut virus in DBT 
cells. (B) IFN 11 mRNA levels in WT- and DUBmut-infected BMDMs were assessed at indicated time points by qRT-PCR. (C) IFN $\alpha$ protein levels in the supernatants of infected BMDMs were evaluated at the times indicated. (D) Comparison of IFNa11 levels in B6 versus MDA5-/- BMDMs at 12 hours post-infection (HPI). (E) Assessing levels of viral nucleocapsid (N) mRNA by qRTPCR. (F) Replication kinetics of WT and DUBmut virus in BMDM cells. Data are representative of at least two independent experiments and are presented as means \pm SD. Data in (B) and (C) were statistically analyzed using unpaired t-tests. ${ }^{*}, \mathrm{p}<0.05 ; * *, \mathrm{p}<0.01$.

Figure 5. Evaluating replication and pathogenesis of MHV-DUBmut in mice. Four- (A) or six-week-old (B) mice were infected with indicated doses of MHV. Viral titer in livers and spleens isolated from WT- or DUBmut virus-infected mice was determined by plaque assay. The number of mice in each group is shown in parentheses. Data were statistically analyzed using unpaired ttests and are presented as means \pm SEM. (C) H\&E staining of liver sections from infected mice at 3 and 5 days post-infection (DPI). Representative MHV-associated liver lesions are indicated by arrows.

Figure 6. Alignment of the nCoV-2019 PLpro domain with the X-ray structure of the closely related SARS-CoV PLpro domain in complex with ubiquitin. (A) X-ray structure of SARSCoV PLpro-ubiquitin-aldehyde complex (blue) (PDB: 4MM3) with each of its domains labeled as finger, palm, thumb, and Ubl2. Ubiquitin-aldehyde is colored yellow. The nCoV-2019 PLpro structure (cyan) was modeled by first mutating the residues of SARS PLpro in the X-ray structure to those of nCoV-2019 PLpro based upon the sequence alignment in Figure 1E. The nCoV-2019 PLpro-Ubiquitin-aldehyde complex was then refined using the structure-factor amplitudes and initial phases of the SARS PLP-Ubiquitin aldehyde complex (PDB: 4MM3). The residues that are different between SARS-CoV PLpro and nCoV-2019 PLpro are highlighted as sticks. (B) Potential 
607 interactions between L1803 of SARS-CoV PLpro and nCoV-2019 PLpro with residue A46 of 608 ubiquitin. (C) Predicted interaction between E1772 of SARS-CoV PLpro and nCoV-2019 PLpro 609 and residues R42 and R72 of ubiquitin. (D) Potential interactions between residues I44, V70, and 610 R42 of ubiquitin with residues M1812 of SARS-CoV PLpro and nCoV-2019 PLpro. 


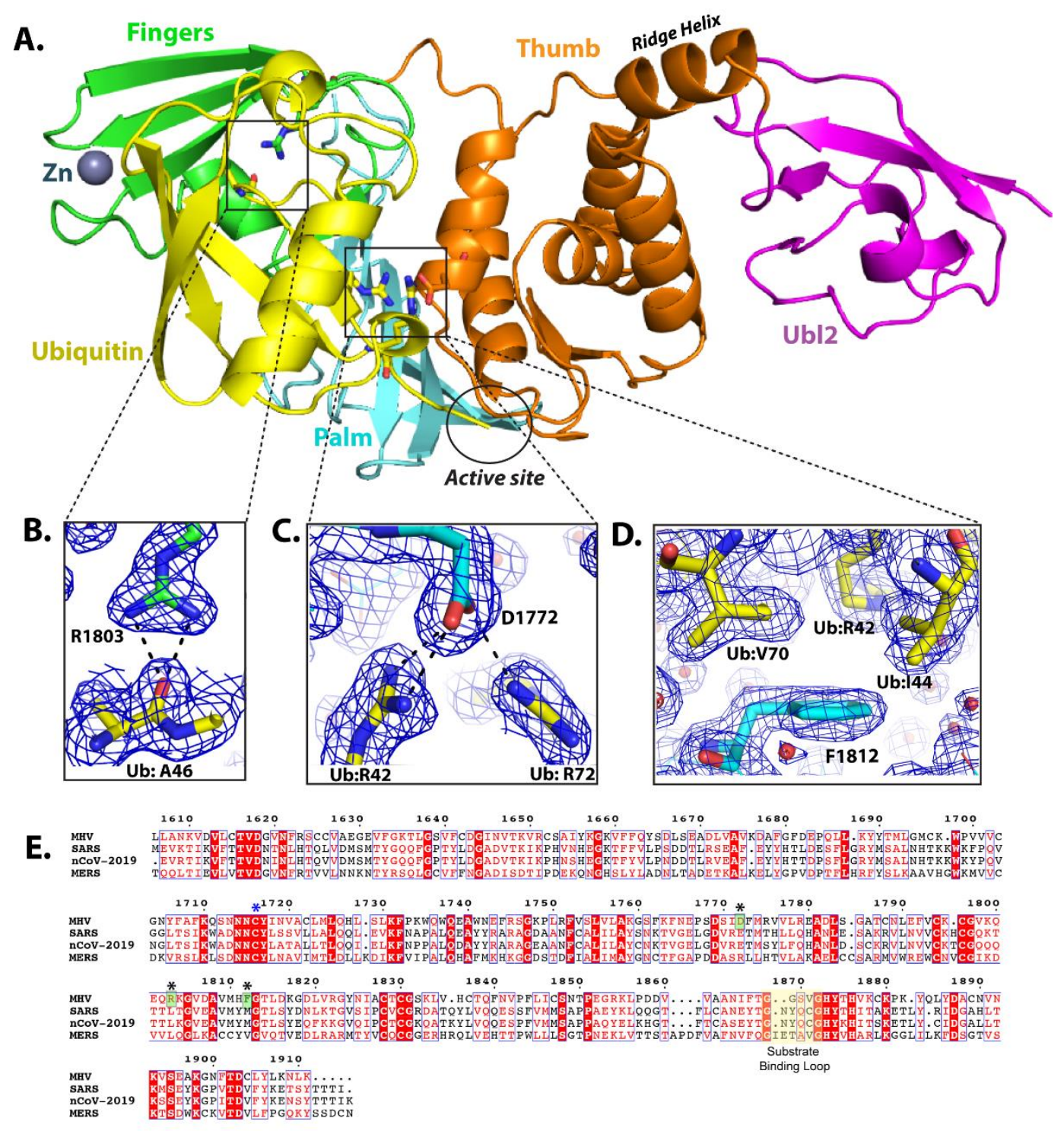

Figure 1. X-ray structure of the MHV PLP2-Ubiquitin complex and residues involved in ubiquitin-binding. (A) Overall structure of MHV PLP2-C1716S-Ub complex. Domains are color coded: Ub in yellow, Ubl2 domain of PLP2 in purple, thumb domain in orange, palm domain in cyan, and fingers domain in green. Residue D1772 of PLP2 is located outside of the active site which is circled in black. (B) Hydrogen bond interactions between MHV PLP2 R1803 and the backbone of A46 of ubiquitin. (C) Binding interactions between MHV PLP2 D1772 and two arginine residues (R42 and R72) of ubiquitin. (D) Hydrophobic interactions between F1812 of MHV PLP2 and ubiquitin residues 144 and V70. The 2Fo-Fc maps (blue) surrounding the residues are contoured at $1 \sigma$ in each panel. The PDB coordinates for the MHV PLP2-C1716S-Ub complex have been deposited under PDB Code 5WFI. (E) Sequence alignment (MultAlign) of coronavirus papain-like protease/deubiquitinating domains from MHV (1606-1911 aa, accession \#AAX23975), SARS (1541-1854 aa, accession \#ACZ72209), 2019-nCoV (1564-1878, accession \#QHO60603) and MERS (1480-1803 aa, accession \# AHY21467). Amino acids are colored by similarity using the RISER coloring scheme. Numbering shown is based on MHV sequence. Amino acids mutated in this study are indicated with a black asterisk, the catalytic cysteine is indicated by a blue asterisk, and those amino acids that bind ubiquitin and were mutated in this study are boxed in green. The active site substrate binding loop also involved in binding inhibitors of SARS is shown highlighted in yellow. The sequence alignment was created using ESPript3. 
A

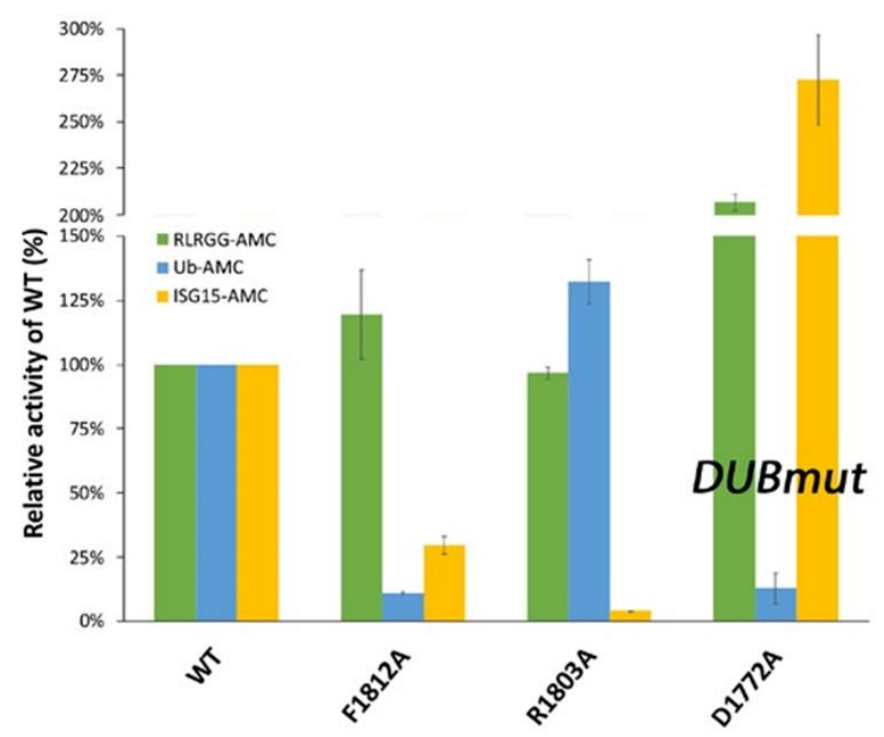

B

\begin{tabular}{l|c|c|c|}
\hline & RLRGG-AMC & Ub-AMC & ISG15-AMC \\
\hline Wild type & & & \\
$K_{\text {cat }}\left(\mathrm{min}^{-1}\right)$ & - & $42.4 \pm 1.2$ & - \\
$\mathrm{K}_{\mathrm{m}}(\mu \mathrm{M})$ & - & $0.67 \pm 0.06$ & - \\
$K_{\mathrm{cat}} / \mathrm{K}_{\mathrm{m}}\left(\mathrm{min}^{-1} \mu \mathrm{M}^{-1}\right)$ & ${ }^{\mathrm{a}} 2.4 \pm 0.04 \times 10^{-3}$ & $63 \pm 5.6$ & ${ }^{2} 2.4 \pm 0.07$ \\
\hline$D 1772 A$ & & & \\
$k_{\mathrm{cat}}\left(\mathrm{min}^{-1}\right)$ & - & - & - \\
$\mathrm{K}_{\mathrm{m}}(\mu \mathrm{M})$ & - & - & - \\
$k_{\mathrm{cat}} / \mathrm{K}_{\mathrm{m}}\left(\mathrm{min}^{-1} \mu \mathrm{M}^{-1}\right)$ & ${ }^{\mathrm{a}} 5.1 \pm 0.07 \times 10^{-3}$ & ${ }^{\mathrm{a}} 7.8 \pm 0.2$ & ${ }^{\mathrm{a}} 7.4 \pm 0.06$ \\
\hline
\end{tabular}

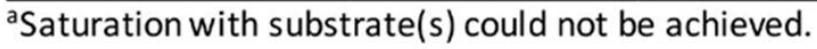

C

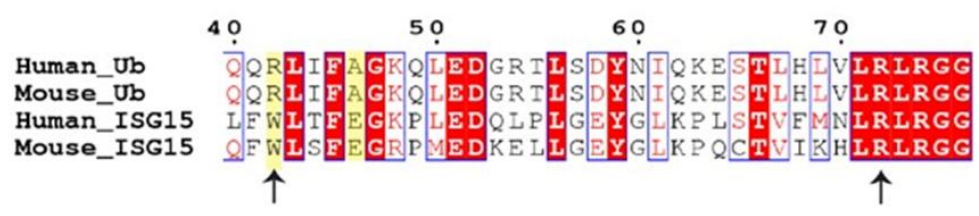

Figure 2. Structure-guided mutagenesis of MHV PLP2 reveals that D1772A disrupts ubiquitin binding and reduces DUB activity. (A) Relative kinetic activities of three mutant MHV PLP2 enzymes toward three substrates: z-RLRGG-AMC (green), Ub-AMC (blue), and ISG15-AMC (yellow) compared to the wild-type enzyme. (B) Steady-state kinetic parameters for wild type and D1772A mutant enzymes. (C) Sequence alignment of Ub and ISG15 from human and mouse generated by Clustal Omega. The two arginine residues of Ub (R42 and R72) that interact with D1772 are indicated by arrows. R72 is conserved between Ub and ISG15, whereas R42 (shaded in yellow) is only present in Ub. Accession numbers: Human_Ub, 1ubq; Mouse_Ub, P62991; Human_ISG15, AAH09507; Mouse_ISG15, AAI09347. The sequence alignment was created using ESPript. 
A

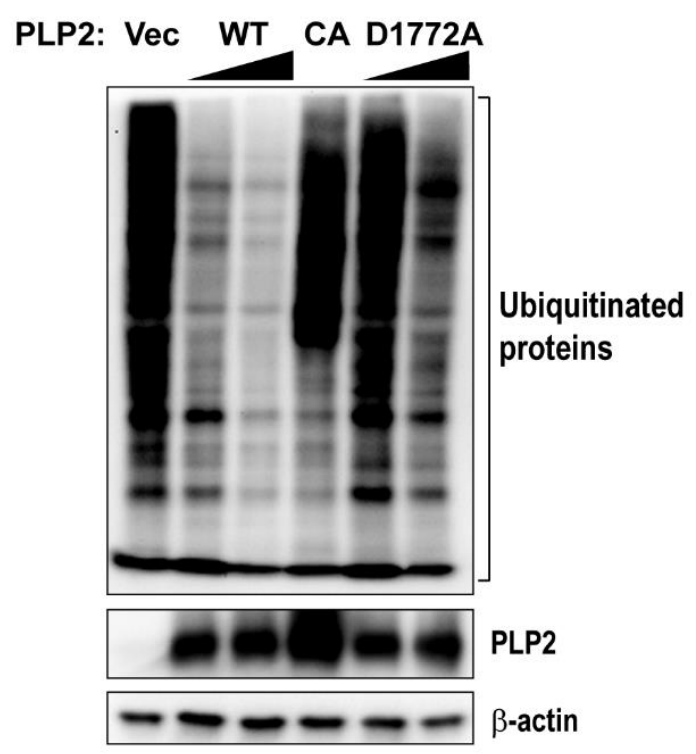

B

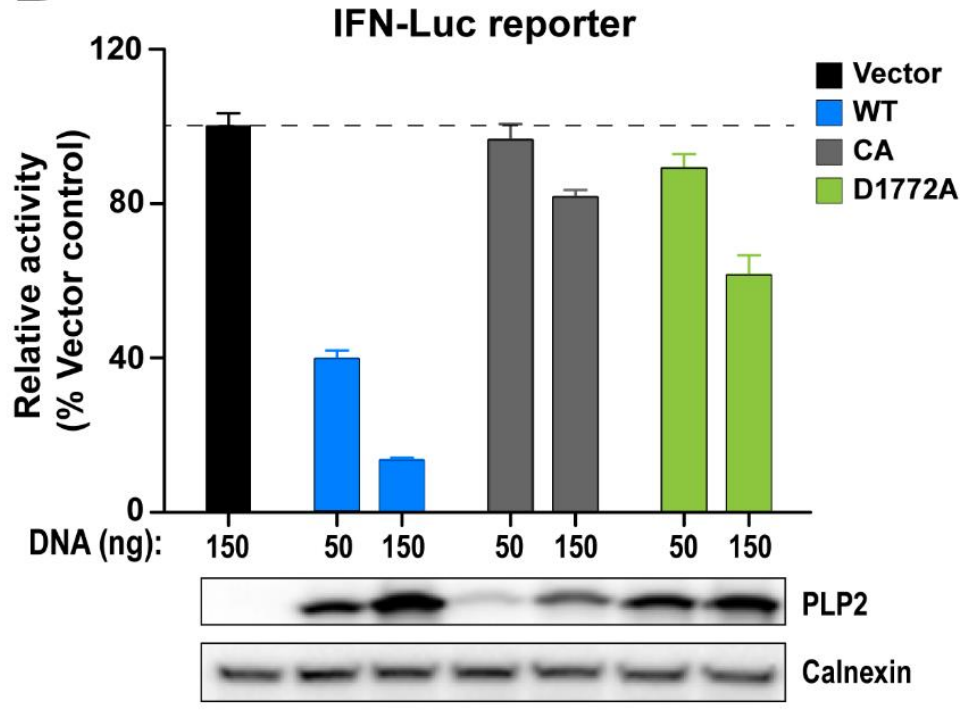

C

D
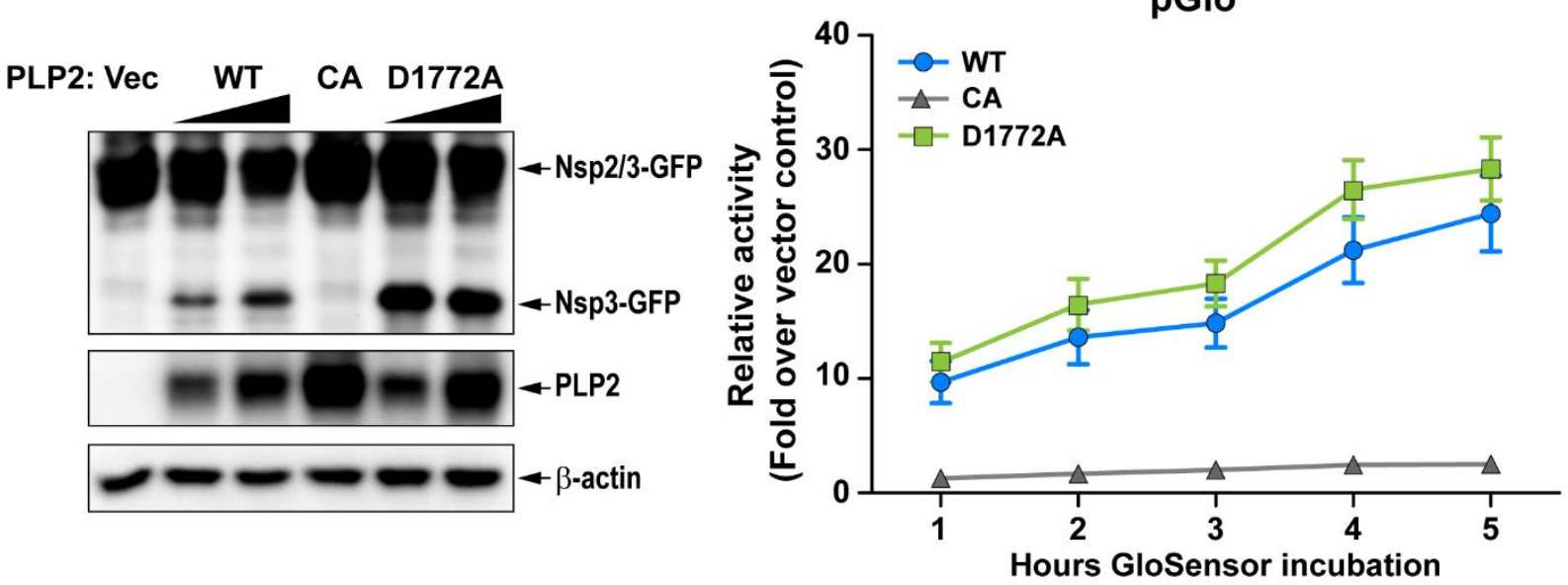

Figure 3. D1772A substitution in the coronavirus papain-like protease Ub-binding site reduces DUB activity and interferon antagonism without reducing protease activity. (A) Western blot assessing the DUB activity of PLP2. (B) IFN antagonism of PLP2 was determined using an IFN-luciferase reporter stimulated by N-RIG-I expression. The reporter activity of vector control was set to $100 \%$ (indicated by a dash line). (C and D) Protease activity was evaluated using $(C)$ a trans-cleavage assay that detects the cleaved products by western blot and (D) a pGlo biosensor assay which is activated by PLP2-mediated cleavage of the substrate. Data are representative of at least two independent experiments. Data in (B) and (D) are presented as means \pm SD. 
A
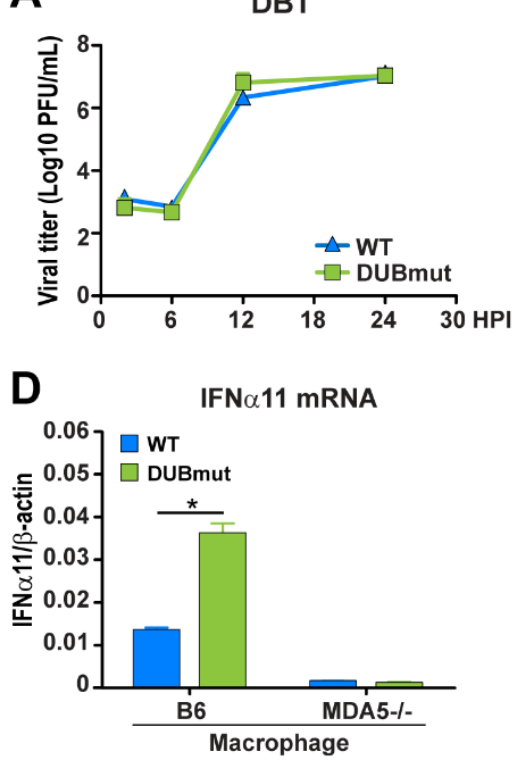

B
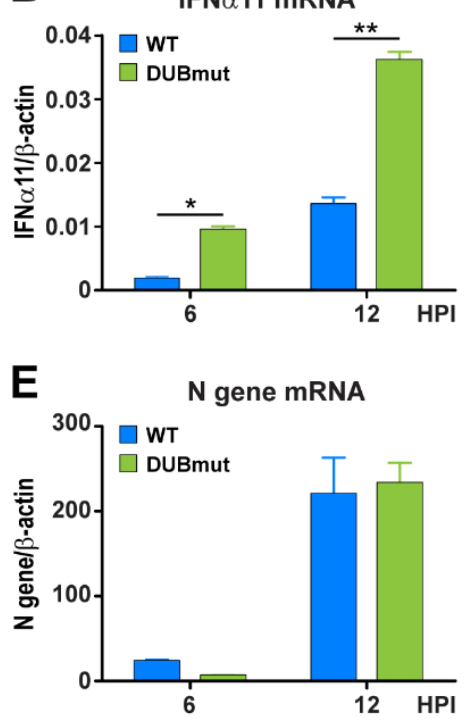

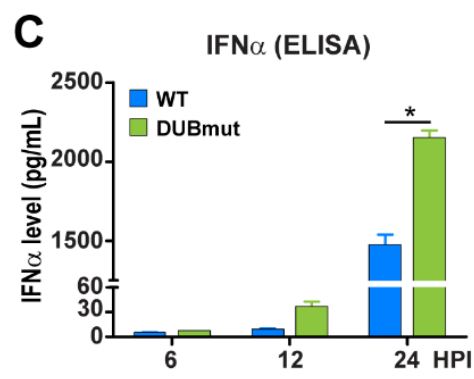

$\mathbf{F}$

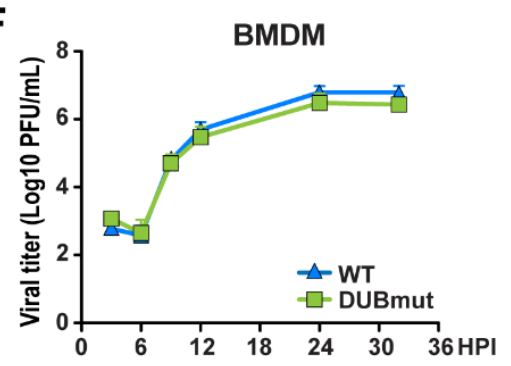

Figure 4. Evaluating the replication kinetics of, and level of interferon activation by, WT MHV and DUBmut in cell culture. (A) Replication kinetics of WT and DUBmut virus in DBT cells. (B) IFNa11 mRNA levels in WT- and DUBmut-infected BMDMs were assessed at indicated time points by qRT-PCR. (C) IFNa protein levels in the supernatants of infected BMDMs were evaluated at the times indicated. (D) Comparison of IFNa11 levels in B6 versus MDA5-/- BMDMs at 12 hours post-infection (HPI). (E) Assessing levels of viral nucleocapsid (N) mRNA by qRT-PCR. (F) Replication kinetics of WT and DUBmut virus in BMDM cells. Data are representative of at least two independent experiments and are presented as means \pm SD. Data in (B) and $(C)$ were statistically analyzed using unpaired t-tests. ${ }^{*}, p<0.05 ;{ }^{* *}, p<0.01$. 
A 4-week-old, $6 \times 10^{3}$ pfu per mouse
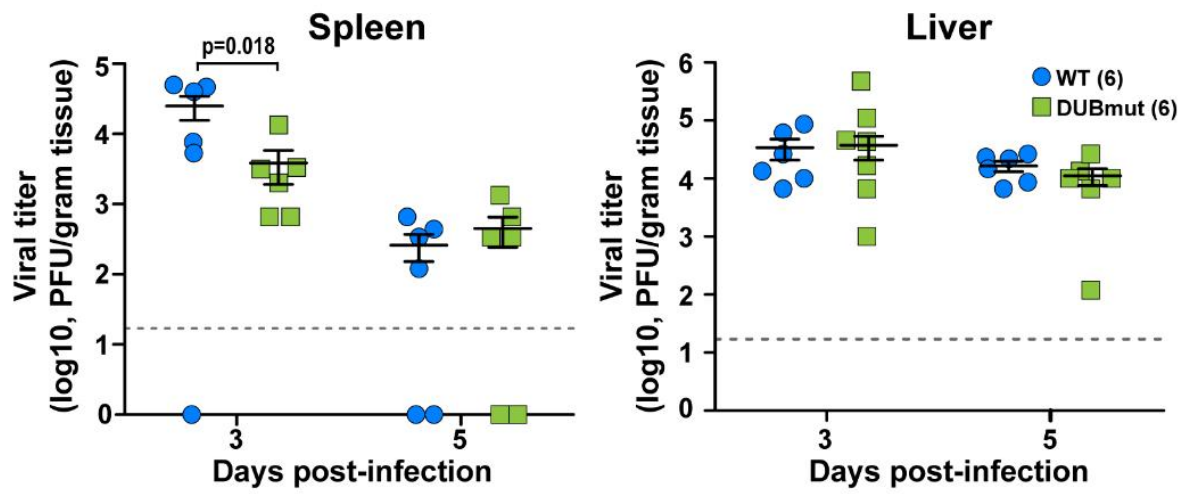

B 6-week-old, $6 \times 10^{4}$ pfu per mouse
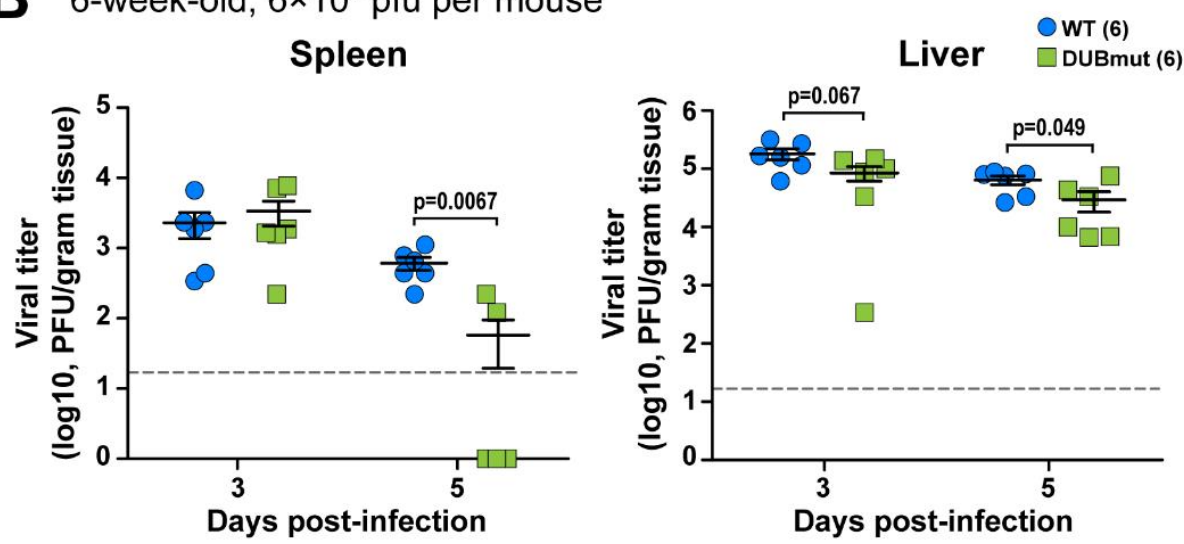

C 6-week-old $6 \times 10^{4}$ pfu per mouse
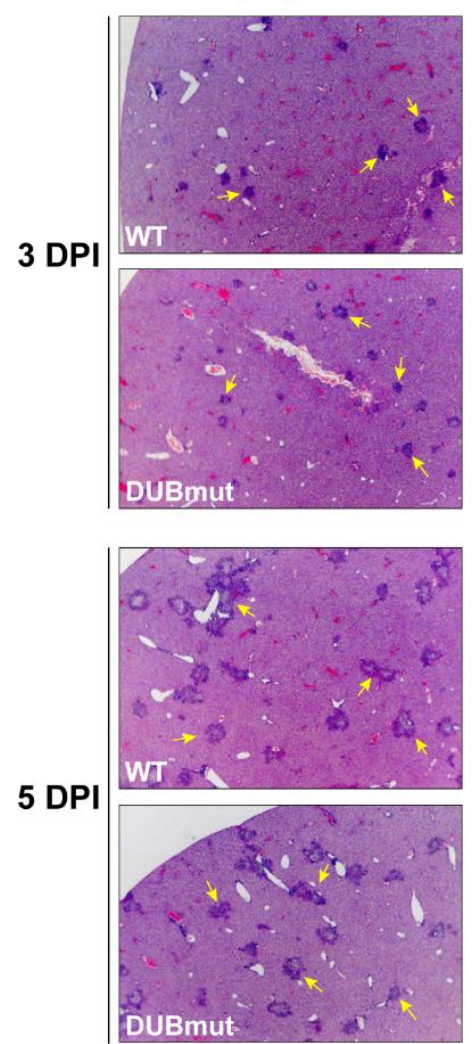

Figure 5. Evaluating replication and pathogenesis of MHV-DUBmut in mice. Four(A) or six-week-old (B) mice were infected with indicated doses of MHV. Viral titer in livers and spleens isolated from WT- or DUBmut virus-infected mice was determined by plaque assay. The number of mice in each group is shown in parentheses. Data were statistically analyzed using unpaired t-tests and are presented as means \pm SEM. (C) $\mathrm{H} \& \mathrm{E}$ staining of liver sections from infected mice at 3 and 5 days post-infection (DPI). Representative MHV-associated liver lesions are indicated by arrows. 


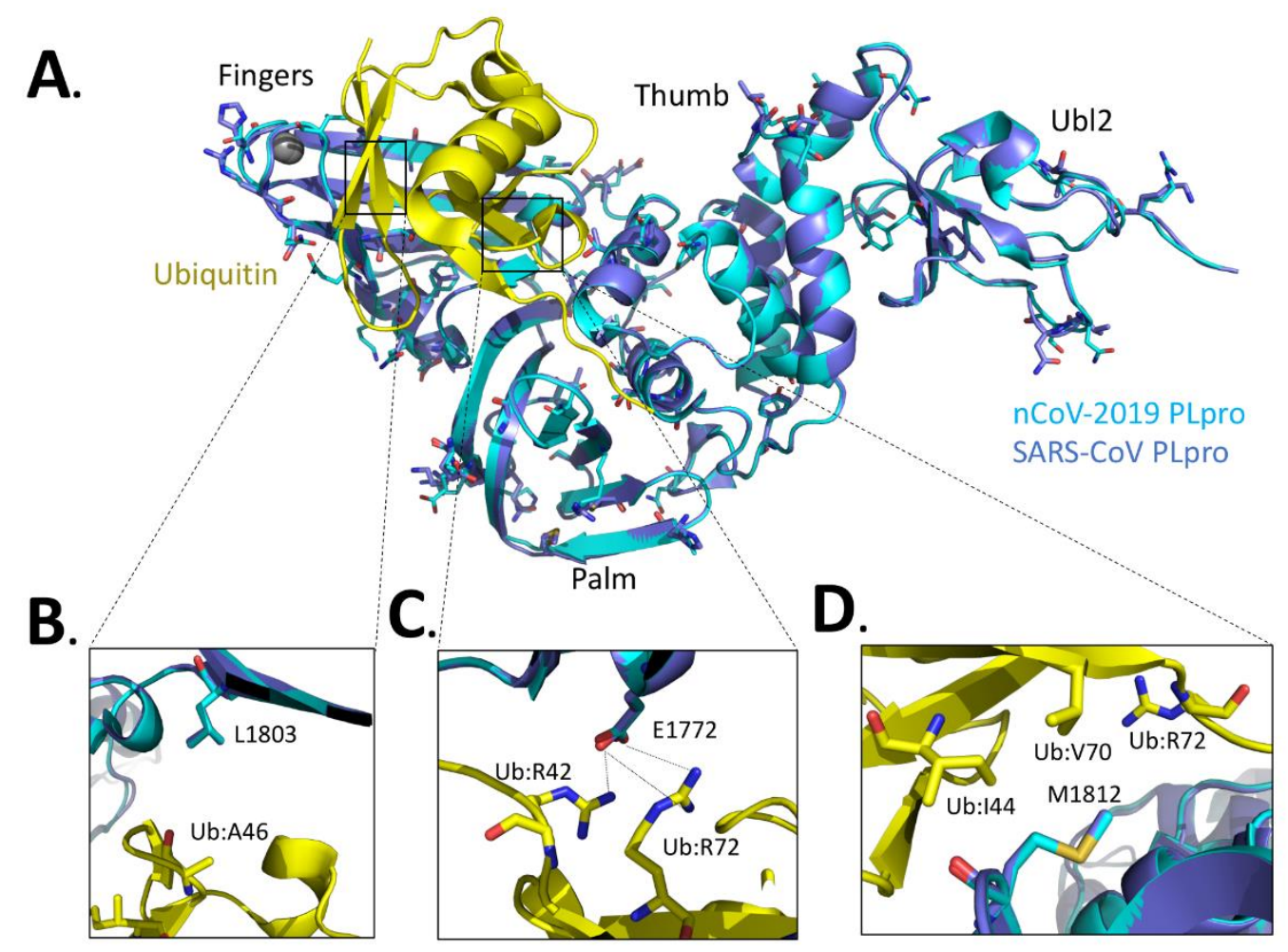

Figure 6. Alignment of the nCoV-2019 PLpro domain with the X-ray structure of the closely related SARS-CoV PLpro domain in complex with ubiquitin. (A) X-ray structure of SARS-CoV PLpro-ubiquitin-aldehyde complex (blue) (PDB: 4MM3) with each of its domains labeled as finger, palm, thumb, and Ubl2. Ubiquitin-aldehyde is colored yellow. The nCoV-2019 PLpro structure (cyan) was modeled by first mutating the residues of SARS PLpro in the X-ray structure to those of nCoV-2019 PLpro based upon the sequence alignment in Figure 1E. The nCoV-2019 PLpro-Ubiquitin-aldehyde complex was then refined using the structure-factor amplitudes and initial phases of the SARS PLP-Ubiquitin aldehyde complex (PDB: 4MM3). The residues that are different between SARS-CoV PLpro and nCoV-2019 PLpro are highlighted as sticks. (B) Potential interactions between L1803 of SARS-CoV PLpro and nCoV-2019 PLpro with residue A46 of ubiquitin. (C) Predicted interaction between E1772 of SARS-CoV PLpro and nCoV-2019 PLpro and residues R42 and R72 of ubiquitin. (D) Potential interactions between residues $144, V 70$, and R42 of ubiquitin with residues M1812 of SARS-CoV PLpro and nCoV-2019 PLpro. 